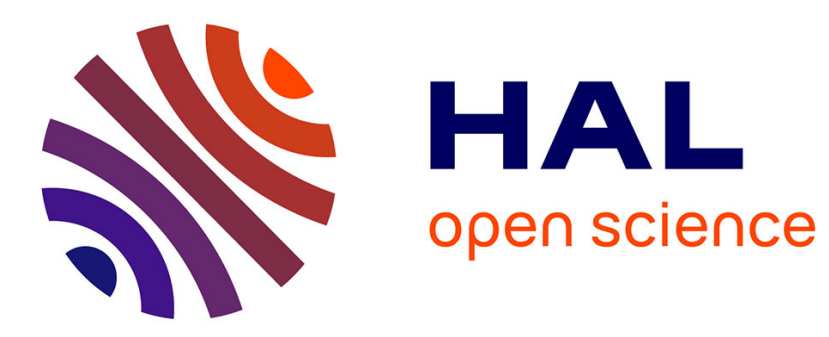

\title{
Renewal theory with a trend
}

Allan Gut

\section{To cite this version:}

Allan Gut. Renewal theory with a trend. Statistics and Probability Letters, 2011, 10.1016/j.spl.2011.03.031 . hal-00753943

\section{HAL Id: hal-00753943 \\ https://hal.science/hal-00753943}

Submitted on 20 Nov 2012

HAL is a multi-disciplinary open access archive for the deposit and dissemination of scientific research documents, whether they are published or not. The documents may come from teaching and research institutions in France or abroad, or from public or private research centers.
L'archive ouverte pluridisciplinaire HAL, est destinée au dépôt et à la diffusion de documents scientifiques de niveau recherche, publiés ou non, émanant des établissements d'enseignement et de recherche français ou étrangers, des laboratoires publics ou privés. 


\section{Accepted Manuscript}

Renewal theory with a trend

Allan Gut

PII:

S0167-7152(11)00122-2

DOI:

10.1016/j.spl.2011.03.031

Reference: STAPRO 5966

To appear in: Statistics and Probability Letters

Received date: 14 October 2010

Revised date: 21 March 2011

Accepted date: 21 March 2011

Please cite this article as: Gut, A., Renewal theory with a trend. Statistics and Probability

Letters (2011), doi:10.1016/j.spl.2011.03.031

This is a PDF file of an unedited manuscript that has been accepted for publication. As a service to our customers we are providing this early version of the manuscript. The manuscript will undergo copyediting, typesetting, and review of the resulting proof before it is published in its final form. Please note that during the production process errors may be discovered which could affect the content, and all legal disclaimers that apply to the journal pertain. 


\title{
Renewal theory with a trend
}

\author{
Allan Gut \\ Uppsala University
}

\begin{abstract}
We prove some analogs of results from renewal theory for random walks in the case when there is a drift, more precisely when the the mean of the $k$ th summand equals $k^{\gamma} \mu, k \geq 1$, for some $\mu>0$ and $0<\gamma \leq 1$.
\end{abstract}

\section{Introduction}

Let $Y, Y_{1}, Y_{2}, \ldots$ be i.i.d. random variables with finite mean 0 and set $X_{k}=Y_{k}+k^{\gamma} \mu$ for $k \geq 1$, and some $\mu>0$. Further, set $T_{n}=\sum_{k=1}^{n} Y_{k}$ and $S_{n}=\sum_{k=1}^{n} X_{k}, n \geq 1$, and define the family of first passage times

$$
\tau(t)=\min \left\{n: S_{n}>t\right\}, \quad t \geq 0
$$

If $\gamma<0$, then

$$
\sum_{k=1}^{n} k^{\gamma}\left\{\begin{array}{lll}
<\infty, & \text { for } \quad \gamma<-1, \\
\sim \log n, & \text { for } \gamma=-1, \\
\sim \frac{1}{\gamma+1} n^{\gamma+1}, & \text { for } \quad-1<\gamma<0
\end{array}\right.
$$

from which it follows that

$$
S_{n}=T_{n}+o(n) \quad \text { as } n \rightarrow \infty,
$$

which means that $\left\{S_{n}, n \geq 1\right\}$ is a perturbed random walk, cf. Gut (2009), Chapter 6 . We therefore assume in the following that $\gamma>0$, and we begin by showing that the stopping times are finite almost surely, and provide conditions for finiteness of moments. For the statements of the latter we introduce the standard notation $x^{+}=\max \{x, 0\}$ and $x^{-}=-\min \{x, 0\}$ for all real $x$.

Set $X_{k}^{\prime}=Y_{k}+\mu, k \geq 1$, and let primed objects refer to this sequence. Since $S_{n}^{\prime} \leq S_{n}$ for all $n$, it follows that

$$
\tau^{\prime}(t) \geq \tau(t) \quad \text { for all } \quad t
$$

and thus, in particular, that $P(\tau(t)<\infty)=1$.

Now, from Gut (1974), Theorem 3.1 (cf. also Gut (2009), Theorem 3.3.1) we know that, for $r \geq 1$

$$
E\left(\tau^{\prime}(t)\right)^{r}<\infty \quad \Longleftrightarrow E\left(X_{1}^{-}\right)^{r}<\infty
$$

This, together with (1.2) and the fact that $E\left(X_{1}^{-}\right)^{r}<\infty \Longleftrightarrow E\left(Y^{-}\right)^{r}<\infty$ establishes the following result.

Theorem 1.1 Let $r \geq 1$. If $E\left(Y^{-}\right)^{r}<\infty$, then $E(\tau(t))^{r}<\infty$.

The analogous result for the stopped sum and the stopping summand turn out as follows.

Theorem 1.2 Let $r \geq 1$. If $E\left(Y^{-}\right)^{(r \gamma) \vee 1}<\infty$ and $E\left(Y^{+}\right)^{r}<\infty$, then $E\left(X_{\tau(t)}\right)^{r}<\infty$ and $E\left(S_{\tau(t)}\right)^{r}<\infty$.

AMS 2000 subject classifications. Primary 60F05, 60F15, 60G50, 60K05; Secondary 60G40.

Keywords and phrases. Trend, renewal theory, first passage time, strong law, central limit theorem, stopping time, elementary renewal theorem.

Abbreviated title. Renewal theory with a trend.

Date. March 21, 2011 
Proof. We first note that

$$
X_{\tau(t)}=X_{\tau(t)}^{+} \leq Y_{\tau(t)}^{+}+(\tau(t))^{\gamma} \mu
$$

where the equality is due to the fact that $P\left(X_{\tau(t)}>0\right)=1$.

Secondly, by Minkowski's inequality and Gut (2009), Lemma 1.8.1 and Remark 1.8.2, we therefore obtain

$$
\left\|X_{\tau(t)}^{+}\right\|_{r} \leq\left\|Y_{\tau(t)}^{+}\right\|_{r}+\left\|(\tau(t))^{\gamma}\right\|_{r} \mu \leq(E \tau(t))^{1 / r} \cdot\left\|Y^{+}\right\|_{r}+\mu\|\tau(t)\|_{\gamma r}^{\gamma},
$$

where $\|\cdot\|_{r}$ denotes the norm of order $r$.

This takes care of the stopping summand.

Integrability of the stopped sum then follows via the "sandwich inequality"

$$
t<S_{\tau(t)} \leq t+X_{\tau(t)}
$$

(cf. Gut (2009), formula (3.3.2)).

REMARK 1.1 The moment condition $E\left(Y^{+}\right)^{r}<\infty$ for the stopped sum is, in fact, necessary, since $S_{\tau(t)} \geq X_{1}^{+} \geq Y_{1}^{+}$.

The first case that comes to mind is the case $\gamma=1$.

Theorem 1.3 For $\gamma=1$ we have

$$
\frac{\tau(t)}{\sqrt{t}} \stackrel{a . s .}{\rightarrow} \sqrt{\frac{2}{\mu}} \quad \text { as } \quad t \rightarrow \infty .
$$

Proof. Since

$$
E S_{n}=\frac{n(n+1) \mu}{2}
$$

the strong law of large numbers tells us that

$$
\frac{S_{n}-\frac{n(n+1) \mu}{2}}{n}=\frac{1}{n} \sum_{k=1}^{n} Y_{k} \stackrel{a . s .}{\rightarrow} 0 \quad \text { as } \quad n \rightarrow \infty,
$$

from which it follows that

$$
\frac{S_{n}}{n(n+1)} \stackrel{\text { a.s. }}{\rightarrow} \frac{\mu}{2}, \quad \frac{S_{n}}{n^{2}} \stackrel{a . s .}{\rightarrow} \frac{\mu}{2}, \quad \frac{X_{n}}{n^{2}} \stackrel{\text { a.s. }}{\rightarrow} 0 \quad \text { as } \quad n \rightarrow \infty .
$$

Since, in view of (1.4), $\tau(t) \nearrow \infty$ a.s. as $t \rightarrow \infty$ we may replace $n$ by $\tau(t)$ (cf. Gut (2009), Theorem 1.2.3(i)), which yields

$$
\frac{S_{\tau(t)}}{(\tau(t))^{2}} \stackrel{a . s .}{\rightarrow} \frac{\mu}{2} \quad \text { and } \quad \frac{X_{\tau(t)}}{(\tau(t))^{2}} \stackrel{a . s .}{\rightarrow} 0 \quad \text { as } \quad n \rightarrow \infty .
$$

With the aid of (1.4) it now follows via "the usual procedure" (cf. Gut (1974), Gut (2009), Chapter 3) that

$$
\frac{t}{(\tau(t))^{2}} \stackrel{a . s .}{\rightarrow} \frac{\mu}{2} \quad \text { as } \quad t \rightarrow \infty .
$$

By combining the theorem with (1.6) the following corollary is immediate.

\section{Corollary 1.1}

$$
\frac{S_{\tau(t)}}{t} \stackrel{\text { a.s. }}{\rightarrow} 1 \quad \text { and } \quad \frac{X_{\tau(t)}}{t} \stackrel{\text { a.s. }}{\rightarrow} 0 \quad \text { as } \quad t \rightarrow \infty .
$$

Note also that there is no central limit theorem for $\tau(t)$ available in this case, since

$$
\frac{X_{n}}{n}=\frac{Y_{n}}{n}+\mu \stackrel{a . s .}{\rightarrow} \mu \neq 0 \quad \text { as } \quad n \rightarrow \infty .
$$

After these introductory results we have reduced the domain of $\gamma$ to the case $0<\gamma<1$, which will be our concern for the remainder of the paper. As we shall see in the following section, there exist a strong law for $\tau(t)$ in this case, a Marcinkiewicz-Zygmund strong law of order $r \in(1,2)$ when $\gamma \in(0,1 / r)$, and a central limit theorem when $\gamma \in(0,1 / 2)$.

Section 3 is devoted to the more general family of first passage times $\tau(t)=\min \left\{n: S_{n}>t \cdot n^{\alpha}\right\}$, $t>0$, where $0<\alpha<1$. A final section contains an analog of the elementary renewal theorem. 
REMARK 1.2 For technical simplicity we confine ourselves the case when the mean and the boundary to be crossed, respectively, increase by powers, and leave the extensions to the cases $X_{k}=Y_{k}+b(k) \mu, k \geq 1$, with $b \in \mathcal{R} \mathcal{V}(\gamma)$ and $\tau(t)=\min \left\{n: S_{n}>t \cdot a(n)\right\}, t \geq 0$, with $a \in \mathcal{R} \mathcal{V}(\alpha)$ to the readers. For the latter case for random walks we refer to Gut (1974) and Gut (2009).

\section{The case $0<\gamma<1$}

Suppose that $Y, Y_{1}, Y_{2}, \ldots$ are i.i.d. random variables with finite mean 0 , and set $X_{k}=Y_{k}+k^{\gamma} \mu$ for some $\mu>0$ and $\gamma \in(0,1)$. Furthermore, let as before, $S_{n}=\sum_{k=1}^{n} X_{k}, n \geq 1$, and define the family of first passage times

$$
\tau(t)=\min \left\{n: S_{n}>t\right), \quad t \geq 0 .
$$

Before we continue, here are some auxiliary facts.

Lemma 2.1 For any $\beta>-1$,

$$
\frac{n^{\beta+1}}{\beta+1} \leq \sum_{k=1}^{n} k^{\beta} \leq \frac{n^{\beta+1}}{\beta+1}+n^{\beta} \quad \text { and } \quad \lim _{n \rightarrow \infty} n^{-(\beta+1)} \sum_{k=1}^{n} k^{\beta}=\frac{1}{\beta+1} .
$$

This result is well known; for a proof, see e.g. Gut (2007), Lemma A.3.1.

Lemma 2.2 Let $1<r<2$ and suppose that $0<\gamma<1 / r$. If $E|Y|^{r}<\infty$, then

$$
\frac{X_{\tau(t)}}{(\tau(t))^{1 / r}} \stackrel{a . s .}{\rightarrow} 0 \quad \text { as } \quad t \rightarrow \infty
$$

Proof. An application of Gut (2009), Theorem 1.2.3(i) tells us that

$$
\frac{X_{\tau(t)}-(\tau(t))^{\gamma} \mu}{(\tau(t))^{1 / r}}=\frac{Y_{\tau(t)}}{(\tau(t))^{1 / r}} \stackrel{a . s .}{\rightarrow} 0 \quad \text { as } \quad t \rightarrow \infty
$$

from which the conclusion follows in view of the fact that $0<\gamma<1 / r$.

After this we turn our attention to the promised limit theorems.

Theorem 2.1 (The strong law)

$$
\frac{\tau(t)}{t^{1 /(\gamma+1)}} \stackrel{a . s .}{\rightarrow}\left(\frac{\gamma+1}{\mu}\right)^{1 /(\gamma+1)} \quad \text { as } \quad t \rightarrow \infty .
$$

Proof. By Lemma 2.1 the strong law in this setting becomes

$$
\frac{S_{n}-\frac{\mu}{\gamma+1} n^{\gamma+1}}{n} \stackrel{a . s .}{\rightarrow} 0 \quad \text { as } \quad n \rightarrow \infty
$$

from which we conclude that

$$
\frac{S_{n}}{n^{\gamma+1}} \stackrel{a . s .}{\rightarrow} \frac{\mu}{\gamma+1} \quad \text { and } \quad \frac{X_{n}}{n^{\gamma+1}} \stackrel{a . s .}{\rightarrow} 0 \quad \text { as } \quad n \rightarrow \infty .
$$

Recalling (1.4) it follows as in the proof of Theorem 1.3 that

$$
\frac{S_{\tau(t)}}{(\tau(t))^{\gamma+1}} \stackrel{a . s .}{\rightarrow} \frac{\mu}{\gamma+1}, \quad \frac{X_{\tau(t)}}{(\tau(t))^{\gamma+1}} \stackrel{a . s .}{\rightarrow} 0, \quad \frac{t}{(\tau(t))^{\gamma+1}} \stackrel{a . s .}{\rightarrow} \frac{\mu}{\gamma+1} \quad \text { as } \quad t \rightarrow \infty .
$$

The following analog of Corollary 1.1 is immediate.

\section{Corollary 2.1}

$$
\frac{S_{\tau(t)}}{t} \stackrel{a . s .}{\rightarrow} 1 \quad \text { and } \quad \frac{X_{\tau(t)}}{t} \stackrel{a . s .}{\rightarrow} 0 \quad \text { as } \quad t \rightarrow \infty
$$


Theorem 2.2 (The Marcinkiewicz-Zygmund strong law) Let $1<r<2$ and $\gamma \in(0,1 / r)$. If $E|Y|^{r}<\infty$, then

$$
\frac{\tau(t)-\left(\frac{(\gamma+1) t}{\mu}\right)^{1 /(\gamma+1)}}{t^{(1-r \gamma) /(r(\gamma+1))}} \stackrel{\text { a.s. }}{\rightarrow} 0 \quad \text { as } \quad t \rightarrow \infty .
$$

Proof. The ordinary Marcinkiewicz-Zygmund (1937) strong law (see also e.g. Gut (2007), Theorem 6.7.1), together with Lemma 2.1, tells us that

$$
\frac{S_{n}-\frac{\mu}{\gamma+1} n^{\gamma+1}}{n^{1 / r}} \stackrel{a . s .}{\rightarrow} 0 \quad \text { as } \quad n \rightarrow \infty
$$

so that, by following the above procedure, we obtain

$$
\frac{S_{\tau(t)}-\frac{\mu}{\gamma+1}(\tau(t))^{\gamma+1}}{(\tau(t))^{1 / r}} \stackrel{\text { a.s. }}{\rightarrow} 0 \quad \text { as } \quad t \rightarrow \infty
$$

and, hence, via (1.4), Lemma 2.2, and Theorem 2.1, that

$$
\frac{t-\frac{\mu}{\gamma+1}(\tau(t))^{\gamma+1}}{t^{1 /(r(\gamma+1))}} \stackrel{a . s .}{\rightarrow} 0 \quad \text { as } \quad t \rightarrow \infty
$$

or, equivalently, that

$$
\left(\frac{(\tau(t))^{\gamma+1}}{t}-\frac{\gamma+1}{\mu}\right) \cdot t^{\rho} \stackrel{a . s .}{\rightarrow} 0 \quad \text { as } \quad t \rightarrow \infty,
$$

where $\rho=1-(r(\gamma+1))^{-1}$.

To finish off we use Taylor expansion (cf. Gut (1974), Gut (2009), Theorem 4.5.5) of the function $g(x)=x^{1 /(\gamma+1)}$ at the point $(\gamma+1) / \mu$ :

$$
\left(\frac{\tau(t)}{t^{1 /(\gamma+1)}}-\left(\frac{\gamma+1}{\mu}\right)^{1 /(\gamma+1)}\right) \cdot t^{\rho}=\left(\frac{(\tau(t))^{\gamma+1}}{t}-\frac{\gamma+1}{\mu}\right) \cdot t^{\rho} \cdot g^{\prime}\left(\theta_{t}\right),
$$

where $\theta_{t}$ lies between $(\tau(t))^{\gamma+1} / t$ and $(\gamma+1) / \mu$ and, by Theorem 2.1 , converges almost surely to the latter.

The proof of the theorem is completed upon observing that the right-hand side of $(2.5)$ converges almost surely to $0 \cdot g^{\prime}((\gamma+1) / \mu)=0$ as $t \rightarrow \infty$ in view of $(2.4)$.

Theorem 2.3 (The central limit theorem) Let $\gamma \in(0,1 / 2)$. If $\operatorname{Var} Y=\sigma^{2}<\infty$, then

$$
\frac{\tau(t)-\left(\frac{(\gamma+1) t}{\mu}\right)^{1 /(\gamma+1)}}{t^{(1-2 \gamma) /(2(\gamma+1))}} \stackrel{d}{\rightarrow} N\left(0, \sigma^{2} \cdot \frac{(\gamma+1)^{(1-2 \gamma) /(\gamma+1)}}{\mu^{3 /(\gamma+1)}}\right) \quad \text { as } \quad t \rightarrow \infty .
$$

Proof. We follow the general pattern of the previous proof, with the central limit theorem replacing the Marcinkiewicz-Zygmund strong law, and Anscombe's theorem replacing Gut (2009), Theorem 1.2.3(i).

By the central limit theorem and Lemma 2.1 we first have

$$
\frac{S_{n}-\frac{\mu}{\gamma+1} n^{\gamma+1}}{\sigma \sqrt{n}} \stackrel{d}{\rightarrow} N(0,1) \quad \text { as } \quad n \rightarrow \infty
$$

so that, by Anscombe's theorem (Anscombe (1952), cf. also Gut (2007), Section 7.3 or Gut (2009), Section 1.3) and Theorem 2.1,

$$
\frac{S_{\tau(t)}-(\tau(t))^{\gamma+1} \mu /(\gamma+1)}{\sigma\left(\frac{(\gamma+1) t}{\mu}\right)^{1 /(2(\gamma+1))}} \stackrel{d}{\rightarrow} N(0,1) \quad \text { as } \quad t \rightarrow \infty .
$$

Proceeding as before, that is, applying (1.4), Lemma 2.2 and some reshuffling, leads to

$$
\left(\frac{\mu}{\gamma+1}\right)^{(2 \gamma+3) /(2(\gamma+1))} \cdot \frac{(\tau(t))^{\gamma+1}-(\gamma+1) t / \mu}{\sigma t^{1 /(2(\gamma+1))}} \stackrel{d}{\rightarrow} N(0,1) \quad \text { as } \quad t \rightarrow \infty .
$$


In order to find the appropriate limit theorem for $\tau(t)$ we exploit the delta-method (cf. e.g. Gut (2007), Section 7.4.1) applied to the function $g(x)=x^{1 /(\gamma+1)}$.

Toward this end we first rewrite (2.7) in a form analogous to (2.4), however, keeping track of constants this time:

$$
\left(\frac{(\tau(t))^{\gamma+1}}{t}-\frac{\gamma+1}{\mu}\right) \cdot t^{(2 \gamma+1) /(2(\gamma+1))} \stackrel{d}{\rightarrow} N\left(0, \sigma^{2}\left(\frac{\gamma+1}{\mu}\right)^{(2 \gamma+3) /(\gamma+1)}\right) \quad \text { as } \quad t \rightarrow \infty .
$$

With $\theta_{t}$ as above we then obtain

$$
\begin{aligned}
\left(\frac{\tau(t)}{t^{1 /(\gamma+1)}}\right. & \left.-\left(\frac{\gamma+1}{\mu}\right)^{1 /(\gamma+1)}\right) \cdot t^{(2 \gamma+1) /(2(\gamma+1))}=\left(\frac{(\tau(t))^{\gamma+1}}{t}-\frac{\gamma+1}{\mu}\right) \cdot t^{(2 \gamma+1) /(2(\gamma+1))} \cdot g^{\prime}\left(\theta_{t}\right) \\
& \stackrel{d}{\rightarrow} N\left(0, \sigma^{2}\left(\frac{\gamma+1}{\mu}\right)^{(2 \gamma+3) /(\gamma+1)} \cdot g^{\prime}\left(\frac{\gamma+1}{\mu}\right)^{2}\right) \quad \text { as } \quad t \rightarrow \infty
\end{aligned}
$$

REMARK 2.1 By putting $\gamma=0$ in our results we rediscover the well-known results from "renewal theory for random walks" in Gut (1974), Section 2; cf. also Gut (2009), Chapter 3.

\section{A curved boundary}

In this section we replace (1.1) by the more general boundary

$$
\tau(t)=\min \left\{n: S_{n}>t \cdot n^{\alpha}\right\}, \quad t \geq 0,
$$

for some $\alpha \in[0,1)$; cf. Gut (1974), Section 3, Gut (2009), Section 4.5. Since the case $\alpha=0$ reduces the setup to the earlier one, we assume in the following that $0<\alpha<1$.

By introducing primed random variables as in the early part of the Introduction, it follows as there (however, cf. Gut (2009), Section 4.5) that $\tau(t)$ is finite almost surely.

As for moments of the stopping time we argue as in Gut (1974), Gut (2009), to obtain the following result.

Theorem 3.1 Let $r \geq 1$. If $E\left(Y^{-}\right)^{r}<\infty$, then $E(\tau(t))^{r}<\infty$.

The analog of Theorem 1.2 follows as above with the modification that we have to replace the sandwich inequality (1.4) by Gut (2009), formula (4.5.14), viz.,

$$
t \cdot(\tau(t))^{\alpha}<S_{\tau(t)} \leq t \cdot(\tau(t))^{\alpha}+X_{\tau(t)} \cdot
$$

Theorem 3.2 Let $r \geq 1$.

(i) If $E\left(Y^{-}\right)^{(r \gamma) \vee 1}<\infty$ and $E\left(Y^{+}\right)^{r}<\infty$, then $E\left(X_{\tau(t)}\right)^{r}<\infty$.

(ii) If $E\left(Y^{-}\right)^{(r(\gamma \vee \alpha)) \vee 1}<\infty$ and $E\left(Y^{+}\right)^{r}<\infty$, then $E\left(S_{\tau(t)}\right)^{r}<\infty$.

Now we are ready for the limit theorems analogous to those of Section 2. The proofs follow the same general pattern, although with some additional technicalities.

Theorem 3.3 (The strong law)

$$
\frac{\tau(t)}{t^{1 /(\gamma+1-\alpha)}} \stackrel{\text { a.s. }}{\rightarrow}\left(\frac{\gamma+1}{\mu}\right)^{1 /(\gamma+1-\alpha)} \quad \text { as } t \rightarrow \infty .
$$

Proof. Applying the sandwich inequality (3.2) to (2.1) yields

$$
\frac{S_{\tau(t)}}{(\tau(t))^{\gamma+1}} \stackrel{a . s .}{\rightarrow} \frac{\mu}{\gamma+1}, \quad \frac{X_{\tau(t)}}{(\tau(t))^{\gamma+1}} \stackrel{a . s .}{\rightarrow} 0, \quad \frac{t \cdot(\tau(t))^{\alpha}}{(\tau(t))^{\gamma+1}} \stackrel{a . s .}{\rightarrow} \frac{\mu}{\gamma+1} \quad \text { as } \quad t \rightarrow \infty .
$$

The usual corollary turns out as follows.

\section{Corollary 3.1}

$$
\frac{S_{\tau(t)}}{t^{(\gamma+1) /(\gamma+1-\alpha)}} \stackrel{a . s .}{\rightarrow}\left(\frac{\gamma+1}{\mu}\right)^{\alpha /(\gamma+1-\alpha)} \quad \text { and } \quad \frac{X_{\tau(t)}}{t^{(\gamma+1) /(\gamma+1-\alpha)}} \stackrel{a . s .}{\rightarrow} 0 \quad \text { as } \quad t \rightarrow \infty .
$$


Theorem 3.4 (The Marcinkiewicz-Zygmund strong law) Let $1<r<2$ and $0<\gamma<1 / r$. If $E|Y|^{r}<\infty$, then

$$
\frac{\tau(t)-\left(\frac{(\gamma+1) t}{\mu}\right)^{1 /(\gamma+1-\alpha)}}{t^{(1-r \gamma) /(r(\gamma+1-\alpha))}} \stackrel{\text { a.s. }}{\rightarrow} 0 \quad \text { as } \quad t \rightarrow \infty .
$$

Proof. We proceed as in the proof of Theorem 2.2. Combining (2.3), (3.2), Lemma 2.2 (which remains true also in the present setting), and Theorem 3.3, tells us that

$$
\frac{t \cdot(\tau(t))^{\alpha}-\frac{\mu}{\gamma+1}(\tau(t))^{\gamma+1}}{t^{1 /(r(\gamma+1-\alpha))}} \stackrel{a . s .}{\rightarrow} 0 \quad \text { as } \quad t \rightarrow \infty .
$$

Rewriting this as

$$
\left(\frac{\tau(t)}{t^{1 /(\gamma+1-\alpha)}}\right)^{\alpha} \cdot\left(\frac{(\tau(t))^{\gamma+1-\alpha}}{t}-\frac{\gamma+1}{\mu}\right) \cdot t^{\rho} \stackrel{a . s .}{\rightarrow} 0 \quad \text { as } \quad t \rightarrow \infty,
$$

where $\rho=\frac{r \gamma+r-1}{r(\gamma+1-\alpha)}$, we obtain, via an application of Theorem 3.3 to the first factor, that

$$
\left(\frac{(\tau(t))^{\gamma+1-\alpha}}{t}-\frac{\gamma+1}{\mu}\right) \cdot t^{\rho} \stackrel{a . s .}{\rightarrow} 0 \quad \text { as } \quad t \rightarrow \infty .
$$

To finish off, Taylor expansion of the function $g(x)=x^{1 /(\gamma+1-\alpha)}$ at the point $(\gamma+1) / \mu$ yields

$$
\left(\frac{\tau(t)}{t^{1 /(\gamma+1-\alpha)}}-\left(\frac{\gamma+1}{\mu}\right)^{1 /(\gamma+1-\alpha)}\right) \cdot t^{\rho}=\left(\frac{(\tau(t))^{\gamma+1-\alpha}}{t}-\frac{\gamma+1}{\mu}\right) \cdot t^{\rho} \cdot g^{\prime}\left(\theta_{t}\right)
$$

where $\theta_{t}$ lies between $(\tau(t))^{\gamma+1-\alpha} / t$ and $(\gamma+1) / \mu$ and, via Theorem 3.3, converges almost surely to the latter. The conclusion now follows as before.

Theorem 3.5 (The central limit theorem) Let $0<\gamma<1 / 2$. If $\operatorname{Var} Y=\sigma^{2}<\infty$, then

$$
\frac{\tau(t)-\left(\frac{(\gamma+1) t}{\mu}\right)^{1 /(\gamma+1-\alpha)}}{t^{(1-2 \gamma) /(2(\gamma+1-\alpha))}} \stackrel{d}{\rightarrow} N\left(0, \sigma^{2} \cdot \frac{1}{(\gamma+1-\alpha)^{2}}\left(\frac{\gamma+1}{\mu}\right)^{(3-2 \alpha) /(\gamma+1-\alpha)}\right) \quad \text { as } \quad t \rightarrow \infty .
$$

Proof. The analogs of (2.6) and (2.7) turn out as

$$
\frac{S_{\tau(t)}-(\tau(t))^{\gamma+1} \mu /(\gamma+1)}{\sigma\left(\frac{(\gamma+1) t}{\mu}\right)^{1 /(2(\gamma+1-\alpha))}} \stackrel{d}{\rightarrow} N(0,1) \quad \text { as } \quad t \rightarrow \infty
$$

and

$$
\left(\frac{\mu}{\gamma+1}\right)^{(2 \gamma+3-2 \alpha) /(2(\gamma+1-\alpha))} \cdot \frac{(\tau(t))^{\gamma+1}-\frac{(\gamma+1) t}{\mu}(\tau(t))^{\alpha}}{\sigma t^{1 /(2(\gamma+1))}} \stackrel{d}{\rightarrow} N(0,1) \quad \text { as } \quad t \rightarrow \infty,
$$

respectively, which, in analogy with the proof of Theorem 3.4, yields

$$
\left(\frac{\mu}{\gamma+1}\right)^{(2 \gamma+3-4 \alpha) /(2(\gamma+1-\alpha))} \cdot \frac{(\tau(t))^{\gamma+1-\alpha}-(\gamma+1) t / \mu}{\sigma t^{(1-2 \alpha) /(2(\gamma+1-\alpha))}} \stackrel{d}{\rightarrow} N(0,1) \quad \text { as } \quad t \rightarrow \infty .
$$

In order to prepare for the delta-method we rewrite this as

$$
\left(\frac{(\tau(t))^{\gamma+1-\alpha}}{t}-\frac{\gamma+1}{\mu}\right) \cdot t^{(2 \gamma+1) /(2(\gamma+1-\alpha))} \stackrel{d}{\rightarrow} N\left(0, \sigma^{2}\left(\frac{\gamma+1}{\mu}\right)^{(2 \gamma+3-4 \alpha) /(\gamma+1-\alpha)}\right) \quad \text { as } \quad t \rightarrow \infty .
$$

And with $\theta_{t}$ as before we finally obtain

$$
\begin{aligned}
& \left(\frac{\tau(t)}{t^{1 /(\gamma+1-\alpha)}}-\left(\frac{\gamma+1}{\mu}\right)^{1 /(\gamma+1-\alpha)}\right) \cdot t^{(2 \gamma+1) /(2(\gamma+1-\alpha))} \\
& =\left(\frac{(\tau(t))^{\gamma+1-\alpha}}{t}-\frac{\gamma+1}{\mu}\right) \cdot t^{(2 \gamma+1) /(2(\gamma+1-\alpha))} \cdot g^{\prime}\left(\theta_{t}\right) \\
& \quad \stackrel{d}{\rightarrow} N\left(0, \sigma^{2}\left(\frac{\gamma+1}{\mu}\right)^{(2 \gamma+3-4 \alpha) /(\gamma+1-\alpha)} \cdot g^{\prime}\left(\frac{\gamma+1}{\mu}\right)^{2}\right) \quad \text { as } t \rightarrow \infty .
\end{aligned}
$$

REMARK 3.1 By putting $\alpha=0$ in the results of this section we rediscover those from Section 2, and by putting $\gamma=0$ we rediscover results in Gut (1974), Section 3; cf. also Gut (2009), Section 4.5 (with the regularly varying function there being a power; recall Remark 1.2). 


\section{An elementary renewal theorem}

In this section we prove a so-called elementary renewal theorem for the case $\gamma \in(0,1]$ and $\alpha=0$, that is, we determine the asymptotics for the expected value of the first passage time as $t \rightarrow \infty$.

We begin with the following preliminary.

Proposition 4.1 Let $0<\gamma \leq 1$ and recall that $\alpha=0$.

(i) The family $\{\tau(t) / t, t \geq 1\}$ is uniformly integrable and

$$
\frac{E \tau(t)}{t} \rightarrow 0 \quad \text { as } \quad t \rightarrow \infty
$$

(ii) The family $\left\{X_{\tau(t)} / t, t \geq 1\right\}$ is uniformly integrable and

$$
\frac{E X_{\tau(t)}}{t} \rightarrow 0 \quad \text { as } \quad t \rightarrow \infty
$$

(iii) The family $\left\{S_{\tau(t)} / t, t \geq 1\right\}$ is uniformly integrable and

$$
\frac{E S_{\tau(t)}}{t} \rightarrow 1 \quad \text { as } \quad t \rightarrow \infty
$$

Proof. (i): Uniform integrability is an immediate consequence of (1.2) and Lai's theorem Lai (1975), according to which the family $\left\{\tau^{\prime}(t) / t, t \geq 1\right\}$ is uniformly integrable.

Next, since, by Theorem 2.1, $\tau(t) / t \stackrel{\text { a.s. }}{\rightarrow} 0$ as $t \rightarrow \infty$, it follows (via e.g. Gut (2007), Theorem 5.5.2) that $E(\tau(t) / t) \rightarrow 0$ as $t \rightarrow \infty$.

(ii): We begin by invoking Gut (2009), Theorem 1.8.1, in order to conclude that $\left\{Y_{\tau(t)}^{\prime} / t, t \geq 1\right\}$ is uniformly integrable. Moreover, by (i) and domination, the family $\left\{(\tau(t))^{\gamma} / t, t \geq 1\right\}$ is also uniformly integrable. This, together with (1.3) shows that the same holds true for $\left\{X_{\tau(t)} / t, t \geq 1\right\}$ (check e.g. Gut (2007), Theorem 5.4.6).

An appeal to Corollary 2.1 (and Gut (2007), Theorem 5.5.2) finishes that part of the proof. (iii): Since, by (1.4),

$$
1<\frac{S_{\tau(t)}}{t} \leq 1+\frac{X_{\tau(t)}}{t}
$$

it follows, via (ii), that the family $\left\{\left(S_{\tau(t)} / t\right)^{r}, t \geq 1\right\}$ is uniformly integrable, after which moment convergence follows via (4.1) and (ii).

Here is now the elementary renewal theorem. We begin with the slightly simpler case $\gamma=1$.

Theorem 4.1 Let $\gamma=1$ and suppose, in addition, that $E\left(Y^{-}\right)^{2}<\infty$. Then

$$
\frac{E \tau(t)}{\sqrt{t}} \rightarrow \sqrt{\frac{2}{\mu}} \quad \text { as } \quad t \rightarrow \infty .
$$

Proof. Since $\left\{\sum_{k=1}^{n} Y_{k}, n \geq 1\right\}$ is a martingale and $E \tau(t)<\infty$, it follows from the first Wald equation (cf. e.g. Gut (2007), Theorem 10.14.3(i)) that $E\left(\sum_{k=1}^{\tau(t)} Y_{k}\right)=0$. Since $\tau(t)$ is square integrable for all $t$ (Theorem 1.1) we may rewrite this as

$$
E S_{\tau(t)}=\frac{\mu}{2} E \tau(t)(\tau(t)+1) \geq \frac{\mu}{2} E(\tau(t))^{2} \geq \frac{\mu}{2}(E \tau(t))^{2},
$$

or, equivalently, as

$$
\frac{E S_{\tau(t)}}{t} \geq \frac{\mu}{2} \cdot\left(\frac{E \tau(t)}{\sqrt{t}}\right)^{2} .
$$

Now, since, by Proposition 4.1(iii), the LHS converges to 1 as $t \rightarrow \infty$, it follows that

$$
\limsup _{t \rightarrow \infty} \frac{E \tau(t)}{\sqrt{t}} \leq \sqrt{\frac{2}{\mu}} \quad \text { as } \quad t \rightarrow \infty
$$


The "converse" inequality follows from Theorem 2.1 and Fatou's lemma:

$$
\sqrt{\frac{2}{\mu}} \leq \liminf _{t \rightarrow \infty} \frac{E \tau(t)}{\sqrt{t}}
$$

The basis for the proof of the analog for $0<\gamma<1$ is the same, but some additional technicalities appear.

Theorem 4.2 Let $\gamma \in(0,1)$ and suppose, in addition, that $E\left(Y^{-}\right)^{\gamma+1}<\infty$. Then

$$
\frac{E \tau(t)}{t^{1 /(\gamma+1)}} \rightarrow\left(\frac{\gamma+1}{\mu}\right)^{1 /(\gamma+1)} \quad \text { as } \quad t \rightarrow \infty .
$$

Proof. Once again, $\left\{\sum_{k=1}^{n} Y_{k}, n \geq 1\right\}$ is a martingale and $E \tau(t)<\infty$. Since, by Theorem 1.1, $E(\tau(t))^{\gamma+1}<\infty$, the rewriting of Wald's equation becomes

$$
E S_{\tau(t)}=E\left(\sum_{k=1}^{\tau(t)} k^{\gamma} \mu\right) \geq \frac{\mu}{\gamma+1} E(\tau(t))^{\gamma+1}
$$

where the inequality is a consequence of Lemma 2.1.

By dividing both members in (4.2) with $t$ and by arguing as in the previous proof we then obtain

$$
\limsup _{t \rightarrow \infty} \frac{\mu}{\gamma+1}\left(\frac{E \tau(t)}{t^{1 /(\gamma+1)}}\right)^{\gamma+1} \leq \limsup _{t \rightarrow \infty} \frac{E S_{\tau(t)}}{t}=1 \quad \text { as } \quad t \rightarrow \infty .
$$

The lower bound follows from Theorem 2.1 and Fatou's lemma as before.

\section{Acknowledgement}

I wish to thank an anonymous referee for his/her very careful reading of the manuscript.

\section{References}

[1] Anscombe, F.J., (1952). Large sample-theory of sequential estimation. Proc. Cambridge Philos. Soc. $48,600-607$.

[2] Gut, A., (1974). On the moments and limit distributions of some first passage times. Ann. Probability 2, 277-308.

[3] Gut, A., (2007). Probability: A Graduate Course, Corr. 2nd printing. Springer-Verlag, New York.

[4] Gut, A., (2009). Stopped Random Walks, 2nd ed. Springer-Verlag, New York.

[5] Lai, T.L., (1975). On uniform integrability in renewal theory. Bull. Inst. Math. Acad. Sinica 3, 99-105.

[6] Marcinkiewicz, J., Zygmund, A., (1937). Sur les fonctions indépendantes. Fund. Math. 29, 60-90.

Allan Gut, Department of Mathematics, Uppsala University, Box 480, SE-751 06 Uppsala, Sweden;

Email: allan.gut@math.uu.se

URL: http://www.math.uu.se/〜allan 